\title{
A Complexity Analysis and Entropy for Different Data Compression Algorithms on Text Files
}

\author{
Mohammad Hjouj Btoush, Ziad E. Dawahdeh \\ Department of Computer Science, Al-Balqa Applied University, Mutah University, Karak, Jordan \\ Email: hujooj@bau.edu.jo,m_ziad_d@yahoo.com
}

How to cite this paper: Btoush, M.H. and Dawahdeh, Z.E. (2018) A Complexity Analysis and Entropy for Different Data Compression Algorithms on Text Files. Journal of Computer and Communications, 6, 301-315.

https://doi.org/10.4236/jcc.2018.61029

Received: November 21, 2017

Accepted: January 9, 2018

Published: January 12, 2018

Copyright (c) 2018 by authors and Scientific Research Publishing Inc. This work is licensed under the Creative Commons Attribution International License (CC BY 4.0). http://creativecommons.org/licenses/by/4.0/

\begin{abstract}
In this paper, we analyze the complexity and entropy of different methods of data compression algorithms: LZW, Huffman, Fixed-length code (FLC), and Huffman after using Fixed-length code (HFLC). We test those algorithms on different files of different sizes and then conclude that: LZW is the best one in all compression scales that we tested especially on the large files, then Huffman, HFLC, and FLC, respectively. Data compression still is an important topic for research these days, and has many applications and uses needed. Therefore, we suggest continuing searching in this field and trying to combine two techniques in order to reach a best one, or use another source mapping (Hamming) like embedding a linear array into a Hypercube with other good techniques like Huffman and trying to reach good results.
\end{abstract}

\section{Keywords}

Text Files, Data Compression, Huffman Coding, LZW, Hamming, Entropy, Complexity

\section{Introduction}

Data compression has important applications in the areas of data transmission and data storage despite of the large capacity storage devices that are available these days. Hence, we need an efficient way to store and transmit different types of data such as text, image, audio, and video to reduce execution time and memory size [1].

In 1977, Abraham Lempel and Jakob Ziv created the first of what we now call the LZ family of substitution compressors [2]. Lempel-Ziv-Welch (LZW) is a universal lossless data compression algorithm created by Abraham Lempel, Ja- 
cob Ziv, and Terry Welch. LZW is a general compression algorithm capable of working on almost any type of data [3].

The general principle of data compression algorithms on text files is to transform a string of characters into a new string, which contains the same information, but with new length as small as possible. The efficient data compression algorithm is chosen according to some scales like compression size, compression ratio, processing time or speed, and entropy [4].

Data compression is very important in business and data processing, which reduces data volume and cost of saving it [5].

Data compression is necessary in many fields in application data processing and also it is very important in distributed systems and data transfer. Data compression is a part of information theory helping in reducing data redundancy over network [6]. So it is very important to study different data compression algorithms used on text files to find what the efficient algorithm is that reduces data volume with saving quality of data.

\subsection{Definition: Compression Size}

Is the size of the new file in bits after compression is complete?

\subsection{Definition}

Compression ratios a percentage that results from dividing the compression size in bits by the original file size in bits and then multiplying the result by $100 \%$.

\subsection{Definition: Processing Time or Speed}

Is the time in millisecond that we need for each symbol or character in the original file for compression? It results from dividing the time in millisecond that is needed for compressing the whole file by the number of symbols in the original file and scales as millisecond/symbol.

\subsection{Definition: Entropy}

Is the number that results from dividing the compression size in bits by the number of symbols in the original file and scales as bits/symbol?

\subsection{Definition: Symbol Probability}

A probability for each symbol in the original file is calculated by dividing the frequency of this symbol in the original file by the number of the whole symbols in this file.

\subsection{Definition: Hamming Weight}

Is the number of ones in the N-bits (fixed-length) code word [7]?

In Section 2, four different data compression techniques (LZW, Huffman, Fixed-length code (FLC), and Huffman after using Fixed-length code (HFLC)) are reviewed and explained. In Section 3, these techniques are tested on different 
text files with different sizes and the results are tabulated and analyzed. Finally, Section 4 presents the conclusions and future work.

\section{Data Compression Techniques}

In this section, we will give a short review and explanation with an example for each one of the four techniques that we check in this paper. We use, as an example, the following string of characters as input string $\mathrm{S}=$ "/WED/WE/WEE/WEB/WET" in all techniques and see the compress file that results [8] [9]. Note that the results on this example do not represent standard results and not scale the efficient of those techniques but only as an example because the size of the string (file) is very small.

\subsection{LZW}

In 1977, Abraham Lempel and Jakob Ziv created the first of what we now call the LZ family of substitutional compressors. In 1984, Terry Welch modified the LZ78 compressor for implementation in high-performance disk controllers. The result was LZW algorithm that is commonly found today [2].

LZW is a general compression algorithm capable of working on almost any type of data [3]. LZW compression creates a table of strings commonly occurring in the data being compressed, and replaces the actual data with references into the table. The table is formed during compression at the same time at which the data is encoded and during decompression at the same time as the data is decoded [10].

The algorithm is surprisingly simple. LZW compression replaces strings of characters with single codes. It does not do any analysis of the incoming text. Instead, it just adds every new string of characters it sees to a table of strings. Compression occurs when a single code is output instead of a string of characters. It starts with a "dictionary" of all the single character with indexes 0.255 . It then starts to expand the dictionary as information gets sent through. Pretty soon, redundant strings will be coded as a single bit, and compression has occurred [11]. This means codes 0 - 255 refer to individual bytes, while codes 256 - 4095 refer to substrings [11]. By applying LZW algorithm on the example $S$, we get the following see Table 1.

The compression ratio $=144 / 152 \times 100 \%=94.73 \%$ from the original size, it means that it saves $5.27 \%$ in space or storage of the new file. And entropy = $144 / 19=7.578 \mathrm{bits} / \mathrm{symbol}$ instead of $8 \mathrm{bits} /$ symbol in ASCII (where 19 is the number of symbols in the file or string).

The string table fills up rapidly, since a new string is added to the table each time a code is output. In this highly redundant input, 5 code substitutions were output, along with 7 characters. If we were using 9 bit codes for output, the 19 character input string would be reduced to a 13.5 byte output string. Of course, this example was carefully chosen to demonstrate code substitution. In real world examples, compression usually doesn't begin until a sizable table has been built, usually after at least one hundred or so bytes have been read in. 
Table 1. The compression process of LZW ( $=$ /WED/WE/WEE/WEB/WET).

\begin{tabular}{|c|c|c|c|}
\hline Character & Code output & New code & New string \\
\hline$/ \mathrm{W}$ & I & 256 & $/ \mathrm{W}$ \\
\hline E & $\mathrm{W}$ & 257 & WE \\
\hline $\mathrm{D}$ & E & 258 & ED \\
\hline I & $\mathrm{D}$ & 259 & $\mathrm{D} /$ \\
\hline WE & 256 & 260 & $/ \mathrm{WE}$ \\
\hline I & $\mathrm{E}$ & 261 & $\mathrm{E} /$ \\
\hline WEE & 260 & 262 & /WEE \\
\hline$/ \mathrm{W}$ & 261 & 263 & $\mathrm{E} / \mathrm{W}$ \\
\hline EB & 257 & 264 & WEB \\
\hline l & B & 265 & $\mathrm{~B} /$ \\
\hline WET & 260 & 266 & /WET \\
\hline EOF & $\mathrm{T}$ & 2 & $/ \mathrm{W}$ \\
\hline $\begin{array}{c}\text { Total }=152 \\
\text { byte }\end{array}$ & $\begin{array}{c}\text { Compressed } \\
\text { size }=12 \text { string } \\
{ }^{*} 12 \text { byte }=\end{array}$ & & \\
\hline
\end{tabular}

\subsection{Huffman Algorithm}

Huffman algorithm is the oldest and most widespread technique for data compression. It was developed by David A. Huffman in 1952 and used in compression of many type of data such as text, image, audio, and video. It is based on building a full binary tree for the different symbols that are in the original file after calculating the probability for each symbol and put them in descending order. After that, we derive the code words for each symbol from the binary tree, giving short code words for symbols with large probabilities and longer code words for symbols with small probabilities [1]. By applying Huffman algorithm on the example above, we get the descending probabilities shown in Table 2.

Moreover, the binary tree was built as in Figure 1.

Then, we get the code word for each symbol from the binary tree as in Table 3.

The compressed file for this string (file) will be:

$0110001110110000110000001100011000110001101=43$ bits, instead of $198=$ 152 bits in ASCII.

\subsection{Fixed-Length Code (FLC)}

Most of compression text methods are done into an arbitrary fixed-length binary code 8-bit ASCII code, which is called a byte wise basis (character wise basis). Limited research has been done on a bitwise basis instead of the conventional byte wise basis [9]. The new technique: Fixed-length code (FLC) deals with more effective approach for English text source encoding, it is based on transforming the characters in the source text to be compressed onto a new weighted 


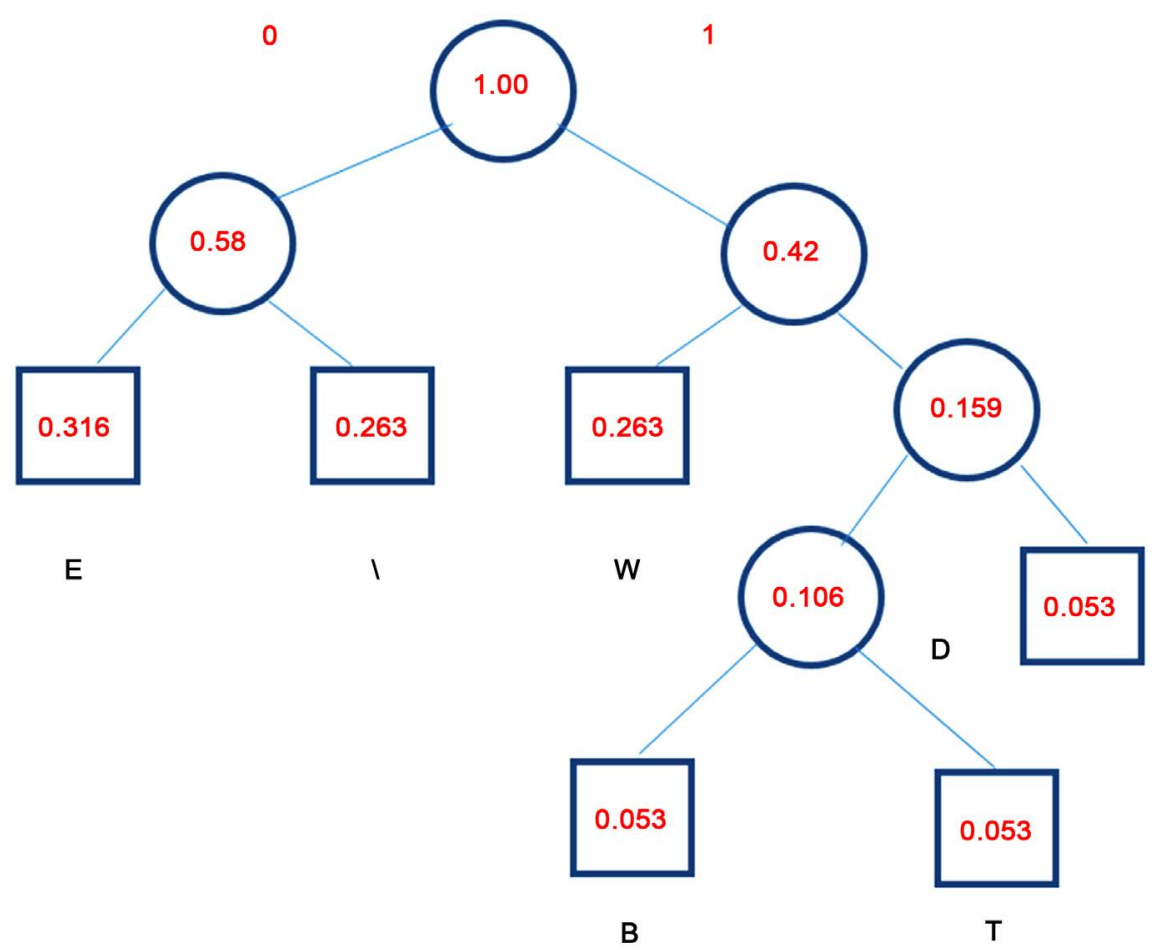

Figure 1. Binary tree for S.

Table 2. Descending probabilities for symbols in S.

\begin{tabular}{cc}
\hline Symbol & Probability \\
\hline E & $6 / 19=0.316$ \\
I & $5 / 19=0.263$ \\
W & $1.15 / 19=0.263$ \\
D & $1 / 19=0.053$ \\
B & $1 / 19=0.053$ \\
T & $1 / 19=0.053$ \\
\hline
\end{tabular}

Table 3. Code words for each symbol in S.

\begin{tabular}{ccc}
\hline Symbol & Probability & Codewords \\
\hline E & 0.316 & 00 \\
/ & 0.263 & 01 \\
W & 0.263 & 10 \\
D & 0.053 & 111 \\
B & 0.053 & 1100 \\
T & 0.053 & 1101 \\
\hline
\end{tabular}

fixed-length binary code by using a bitwise basis (its length depends on the number of different symbols in the source text) rather than a byte wise basis (8-bits ASCII) [6] [10]. Now, we apply this new mapping technique on the ex- 
ample S .First, we calculate the probability for each symbol in the source text and put them in descending order. The length of the new N-bit code word is calculated from $m=2 \mathrm{~N}$, where $\mathrm{m}$ is the number of the different symbols in the source text file and $\mathrm{N}$ is the number of bits (fixed-length) that we need for each character in this text (file) instead of 8-bits. Here $m=6$ symbols, so we need 3-bits $(\mathrm{N}=3)$ for each symbol. The symbol with large probability $(\mathrm{E})$ take a code word with large Hamming weight $(\mathrm{N})$, the next $\left({ }_{\mathrm{r}}^{\mathrm{N}}\right)$ symbols take a code word with (Nr) Hamming weight, and so on (where $\mathrm{r}=1,2, \ldots, \mathrm{N}$ ) [6] [10]. So, we get the results In Table 4.

The compress file by this technique is: $110101111011110101111110101111111110101111100110101111010=57$ bits The compression ratio $=57 / 152 \times 100 \%=37.5 \%$ from the original size, it saves $62.5 \%$ in the space or storage. The entropy for this technique in this file is 3 bits/symbol instead of 8 bits/symbol in ASCII.

\subsection{Huffman after Using Fixed-Length Code (HFLC)}

This technique is a complement to the previous approach. Here, we use Huffman Algorithm on the new fixed-length code (FLC) that we obtained before. First, we calculate the probability of the symbols one and zero from the compressed file that results from the previous technique, then calculate the new probability for each fixed-length code by using the following equation:

$$
\text { New probability }=q^{u}(1-q)^{N-u}
$$

where $u$ is the number of one's in the given fixed-length code, $(N-u)$ is the number of zero's in this code, $\mathrm{q}$ is the probability of the symbol one, and $(1-q)$ is the probability of zero [1]. After that, we apply Huffman algorithm on the new probability that we get after sorting them in descending order and building the full binary tree as we done in Section 2.2. By applying this technique on the results that we get from $S$, the probability for symbol one $=42 / 57=0.737$ and the probability for the symbol zero $=1-0.737=0.263$. The new binary tree is represented by Figure 2 .

The new probability and Huffman code words are illustrated in Table 5.

The compressed file that results from this technique is: $00000110100000011000001110000011011000000110111=47$ bits. The compression ratio $=47 / 152 \times 100 \%=30.92 \%$ from the original size, so it saves $69.08 \%$ of the storage.

Table 4. Codeword for each symbol in FLC.

\begin{tabular}{ccc}
\hline Symbol & Probability & Codeword (FLC) \\
\hline E & 0.316 & 111 \\
I & 0.263 & 110 \\
W & 0.263 & 101 \\
D & 0.053 & 011 \\
B & 0.053 & 100 \\
\hline
\end{tabular}




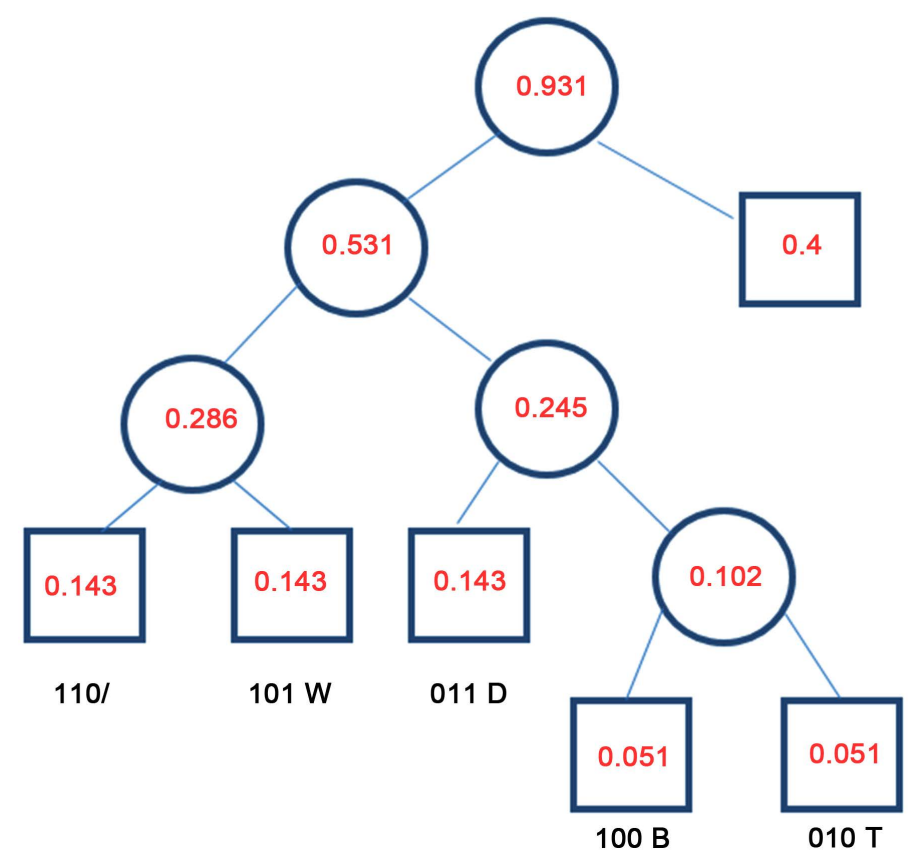

Figure 2. New binary tree.

Table 5. New probability and Huffman code words.

\begin{tabular}{cccc}
\hline Symbol & Fixed-length code & New probability & Huffman code words \\
\hline E & 111 & 0.400 & 1 \\
I & 110 & 0.143 & 000 \\
W & 101 & 0.143 & 001 \\
D & 011 & 0.143 & 010 \\
B & 100 & 0.051 & 0110 \\
T & 010 & 0.051 & 0111 \\
\hline
\end{tabular}

The entropy for this technique on this file is $=47 / 19=2.474$ bits $/$ symbol. Thus, from the results that we got from applying the four techniques (LZW, Huffman, FLC, and HFLC) on the given example, we note that the compression ratios for them are: $94.73 \%, 28.28 \%, 37.5 \%$ and $30.92 \%$, respectively. So, the best one on this example was Huffman, HFLC, FLC, and LZW respectively. We also note that the entropys for these techniques on this example were: 7.578, 2.263, 3.0, and 2.474 bits/symbol, respectively. It is clear that, the best one on this example was Huffman, then HFLC, FLC, and LZW. But we must note that, these results are not standard but only as an example on each one, because LZW gives best results on the big files but its results are worst on the small files see Table A1 (Appendix A), Figure 3 and Figure 4.

Table A2 (Appendix B) shows the files and the compression ratio for each file in each technique, we note that it is high (worst) in LZW for the small files and low (best) in Huffman, but on the big size the best technique is LZW, then Huffman, HFLC, and FLC, respectively. 


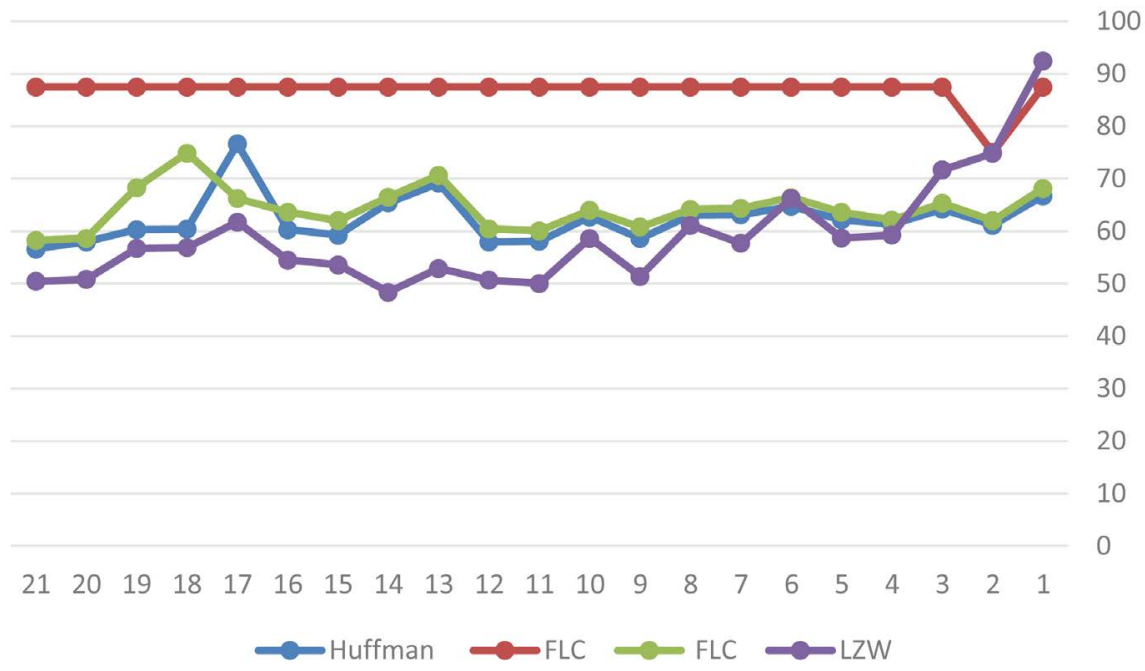

Figure 3. Compression size.

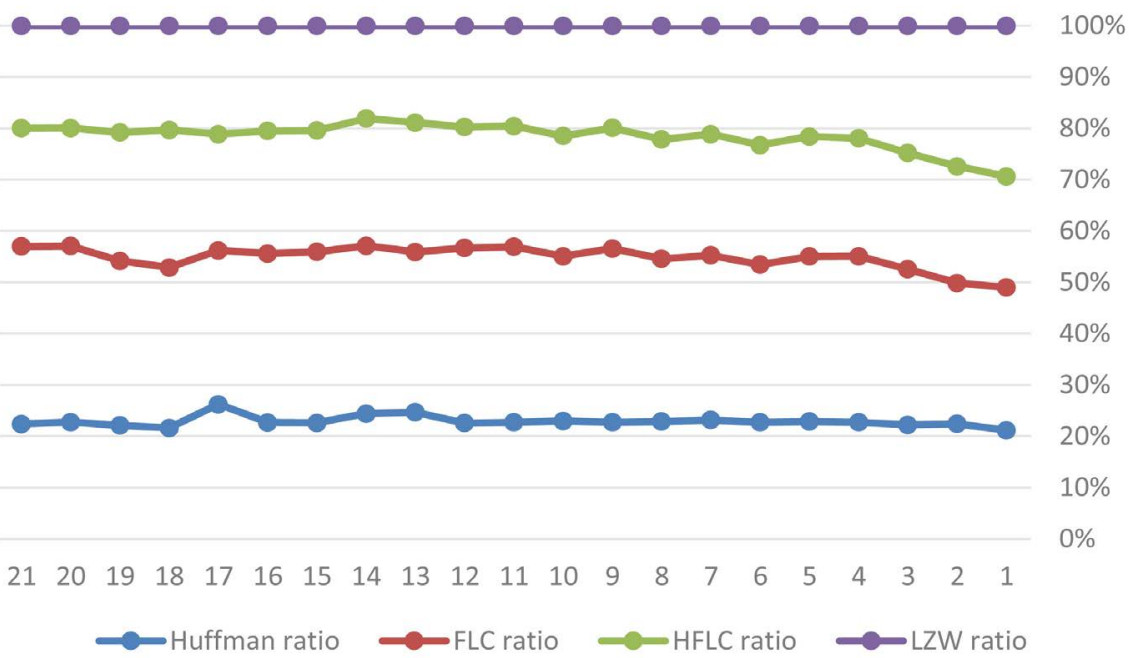

Figure 4. Compression ratio.

\section{Analysis and Results}

In this Section, tests are made on the four types of techniques on different text files (21 files) from different sizes. Some of these files are taken from the Calgary Corpus; which is a set of traditionally files used to test data compression programs [5]. The results are tabulated and analyzed in order to reach to the best technique, advantage and disadvantage for each one, and when each one is best to use. Source code is written for each technique; in C++ for Huffman, FLC, and Huffman after using FLC, and in Java for LZW. The execution for these programs is done on Pentium 4 with $2.4 \mathrm{G}$, Ram $248 \mathrm{M}$, and full cache. The following results are obtained: Table A1 shows tested files names, original size in bytes and bits, and the new size for each file after compression in the four techniques that we tested. It is clear that Huffman Algorithm is the best one on the small files, then HFLC, FLC, and LZW, but when the size of the files increase LZW will be the best one, then Huffman, HFLC, and FLC, respectively. 
Table A2 (Appendix B) shows the files and the compression ratio for each file in each technique, we note that it is high (worst) in LZW for the small files and low (best) in Huffman, but on the big size the best technique is LZW, then Huffman, HFLC, and FLC, respectively.

Table A3 (Appendix C) and Figure 5 represents the compression time in milliseconds that is needed for each character in the source files to complete compression in the four techniques. The best (smallest) time is in LZW, the time in Huffman and FLC is nearly the same, and in HFLC is the worst (long) time because as we saw in the example in Section 2.4, we need more calculations before building the binary tree and obtain the code words.

Now, we illustrate all the results that we obtained from all tested files by taking the average for each of: the original size, the compression size, the compression ratio, the compression time, and the entropy in the four techniques. It is clear from Table A4 (Appendix D) and Table A5 (Appendix E), Figures 6-9 that the average of: compression size, compression ratio, compression time, and entropy is the best in LZW, then in Huffman, HFLC, and FLC, respectively.

From all of the above, LZW is the best technique in all of the compression scales that we tested especially on the files of big sizes, then Huffman, HFLC, and FLC, respectively. But we must note that, the performance of the data compression depends on: the characteristics of the files, the different symbols contained in it, and symbols frequencies. We also note that FLC is a good technique and give a good result when the file contained little different characters or symbols,
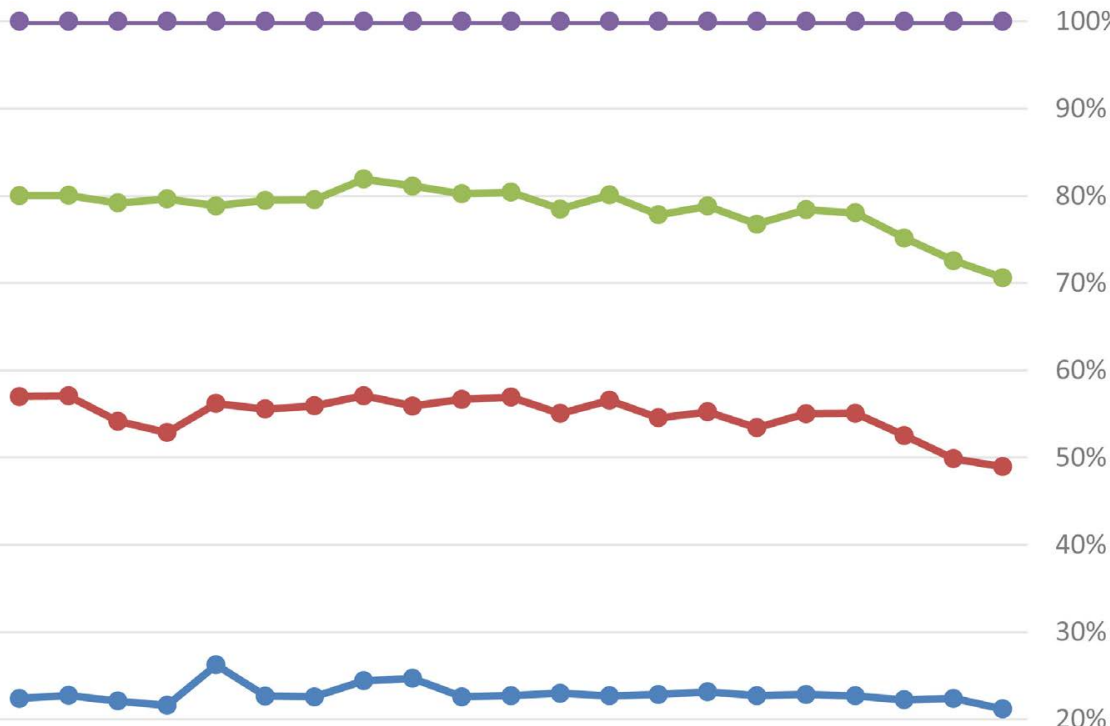

$\begin{array}{lllllllllllllllllllll}21 & 20 & 19 & 18 & 17 & 16 & 15 & 14 & 13 & 12 & 11 & 10 & 9 & 8 & 7 & 6 & 5 & 4 & 3 & 2 & 1\end{array}$

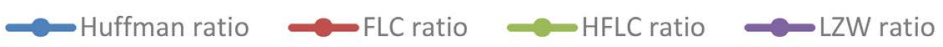

Figure 5. Compression time. 

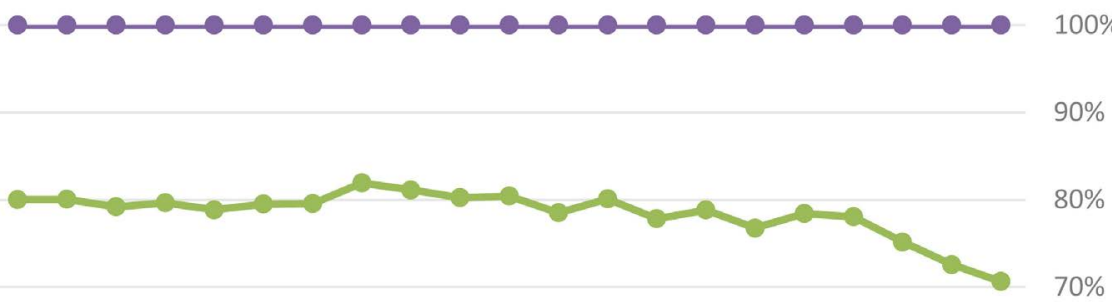

$80 \%$

$70 \%$

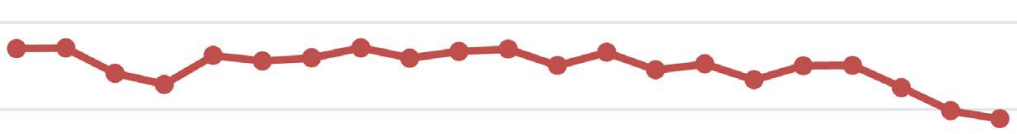

$60 \%$
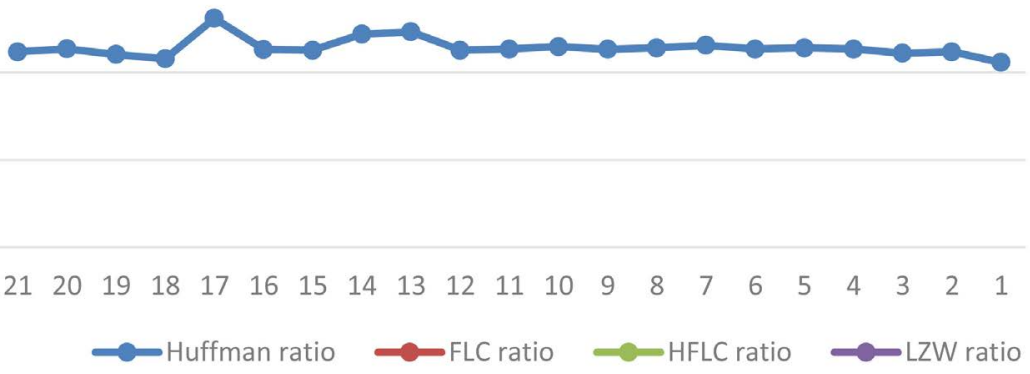

Figure 6. Entropy.

\section{A verage ratio}

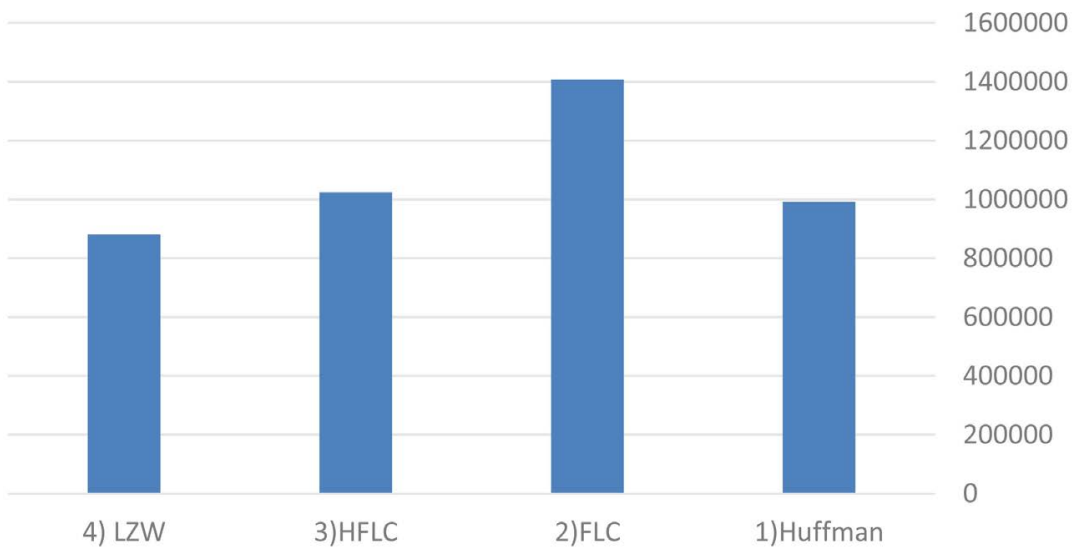

Figure 7. Average ratio.

for example less than 16 different symbols. The advantage of this technique is in the memory space because it deals with 4 bits instead of 8 bits in ASCII for each character. But in fact, we need data compression for large files that contains different characters in order to reduce its size, so this technique will not give a good results on it because if the number of different symbols in the file is more than 64, we will need 7 or 8 bits for each character which is nearly the same as in ASCII, and in this case we need more time for the new calculations that we need in this technique. 


\section{Average compress ratio}

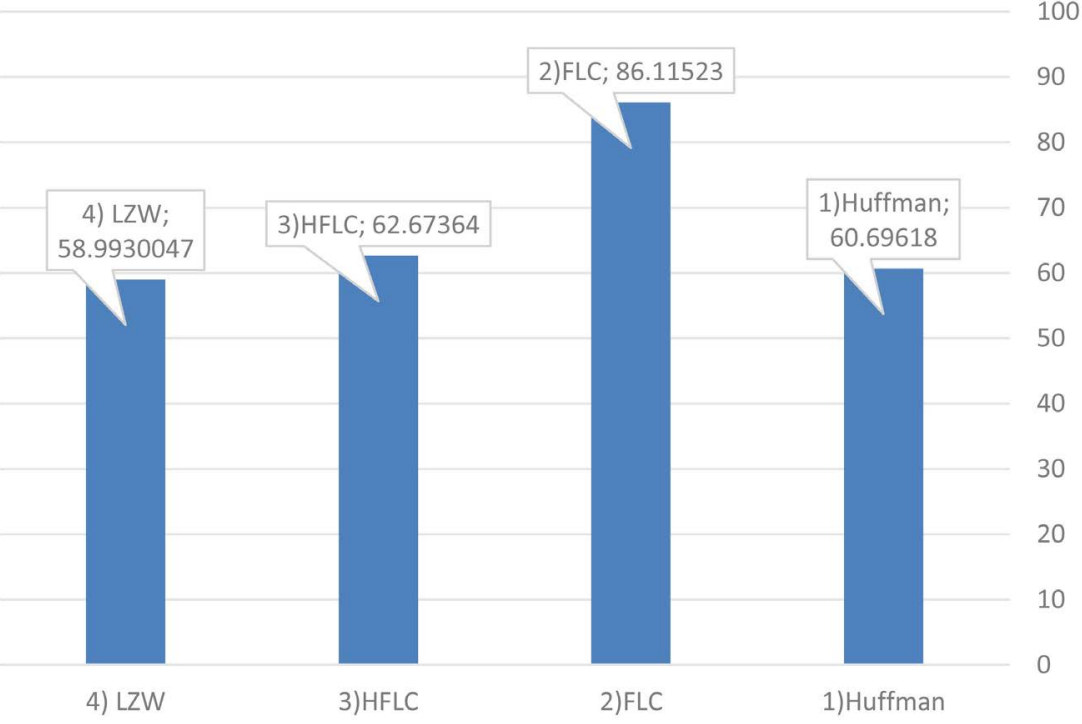

Figure 8. Average compression ratio.

\section{Average entropy \\ bits \char}

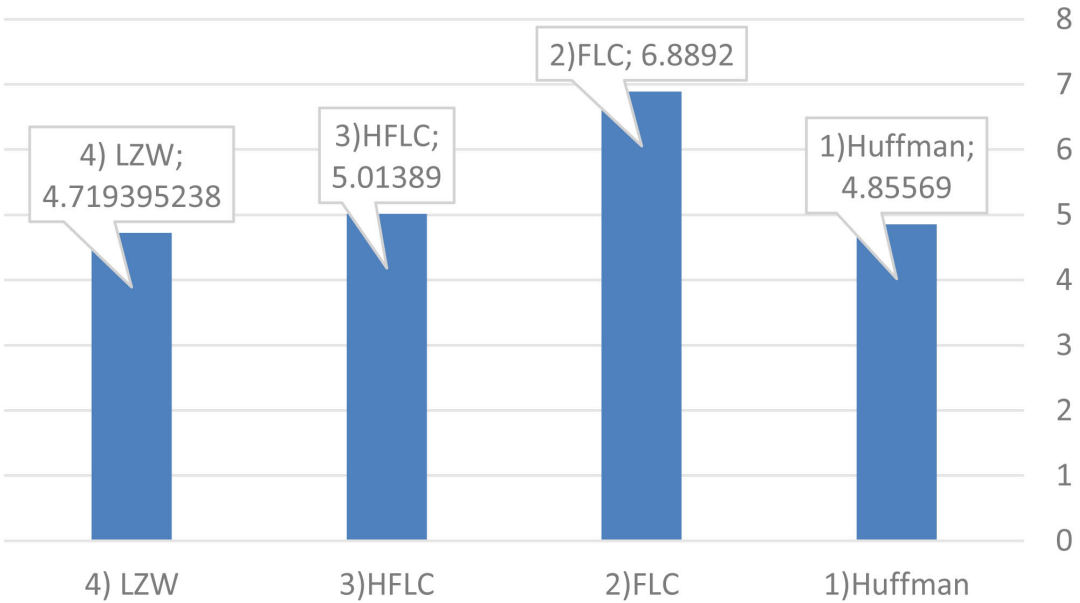

Figure 9. Average entropy.

Another advantage for LZW is that it does not need to pass the large string table to the decompression code, the table can be built exactly as it was during compression [12]. Whereas, in Huffman we must transmit the frequency table for the characters in the source file in order to enable from building the binary tree, which will increase the size of the transmitted (compress) file [13].

\section{Conclusions}

In this paper, we analyze the complexity and entropy of different methods of data compression algorithms: LZW, Huffman, Fixed-length code (FLC), and 
Huffman after using Fixed-length code (HFLC). We test those algorithms on different files of different sizes and then conclude that: LZW is the best one in all compression scales that we tested especially on the large files, then Huffman, HFLC, and FLC, respectively.

From all of the above, LZW is the best technique in all of the compression scales that we tested especially on the files of big sizes, then Huffman, HFLC, and FLC, respectively. But we must note that, the performance of the data compression depends on: the characteristics of the files, the different symbols contained in it, and symbols frequencies.

\section{References}

[1] Elabdalla, A.R. and Irshid, M.I. (2001) An Efficient Bitwise Huffman Coding Technique Based on Source Mapping. Computer and Electrical Engineering, 27, 265-272. https://doi.org/10.1016/S0045-7906(00)00022-7

[2] Cheng, C.Y. (2001) Introduction on Text Compression Using Lempel, Ziv, Welch (LZW) Method. http://www.geocities.com/yccheok/lzw/lzw.html

[3] Heggeseth, M. (2003) Compression Algorithms: Huffman and LZW, CS 372: Data Structures. http://web.mit.edu/6.02/www/s2012/handouts/3.pdf

[4] Lelewer, D.A. and Hirschberg, D.S. (1987) Data Compression. http://www.ics.uci.edu/ dan/pubs/DataCompression.html

[5] Hasan, M.R., et al. (2013) Comparative Data Compression Techniques and Multi-Compression Results. IOP Conference Series: Materials Science and Engineering, 53, 012081. https://doi.org/10.1088/1757-899X/53/1/012081

[6] Sethi, G., et al. (2014) Data Compression Techniques. International Journal of Computer Science and Information Technologies, 5, 5584-5586.

[7] Jaradat, A.R. and Irshid, M.I. (2001) A Simple Binary Run-Length Compression Technique for Non-Binary Source Based on Source Mapping. Active and Passive Electronic Components, 24, 211-221.

[8] Nelson, M. (1989) LZW Data Compression, Dr. Dobb’s Journal. http://www.dogma.net/markn/articles/lzw/lzw.htm

[9] MIT 6.02 DRAFT Lecture Notes (2010) Source Coding: Lossless Compression. http://web.mit.edu/6.02/www/f2010/handouts/lectures/L22.pdf

[10] Ferreira, A.J., Oliveira, A.L. and Figueiredo, M.A.T. (2009) On the Suitability of Suffix Arrays for Lempel-Ziv Data Compression. In: Filipe, J., Obaidat, M.S., Eds., e-Business and Telecommunications. ICETE 2008, Communications in Computer and Information Science, Vol. 48, Springer, Berlin, Heidelberg.

[11] Arimura, M. and Yamamoto, H. (1998) Asymptotic Optimality of the Block Sorting Data Compression Algorithm. IEICE Transactions on Fundamentals, E81-A, 2117-2122.

[12] Salomon, D. (2008) A Concise Introduction to Data Compression. Springer-Verlag, London. http://www.springer.com/gp/book/9781848000711

[13] Nelson, M. (1989) LZW Data Compression. Data Compression, Magazine Articles. http://marknelson.us/1989/10/01/lzw-data-compression/ 


\section{Appendix A}

Table A1. List of files before and after compression.

\begin{tabular}{|c|c|c|c|c|c|c|}
\hline File name & $\begin{array}{l}\text { Original } \\
\text { size } \\
\text { (bytes) }\end{array}$ & $\begin{array}{c}\text { Original } \\
\text { size } \\
\text { (bits) }\end{array}$ & $\begin{array}{c}\text { Huffman } \\
\text { size } \\
\text { (bits) }\end{array}$ & $\begin{array}{c}\text { FLC } \\
\text { size } \\
\text { (bits) }\end{array}$ & $\begin{array}{l}\text { HFLC } \\
\text { size } \\
\text { (bits) }\end{array}$ & $\begin{array}{c}\text { LZW } \\
\text { size } \\
\text { (bits) }\end{array}$ \\
\hline 1) test 1.txt & 1024 & 8192 & 5463 & 7168 & 5585 & 7576 \\
\hline 2) test 2.txt & 2048 & 16,384 & 10,016 & 12,288 & 10,162 & 12,264 \\
\hline 3) test 3.txt & 4096 & 32,768 & 21,031 & 28,672 & 21,407 & 23,488 \\
\hline 4) test 4.txt & 8192 & 65,536 & 40,199 & 57,344 & 40,700 & 38,848 \\
\hline 5) paper 5.txt & 11954 & 95,632 & 59,445 & 83,678 & 60,819 & 56,136 \\
\hline 6) test 5.txt & 16384 & 131,072 & 84,815 & 114,688 & 87,073 & 86,800 \\
\hline 7) test 6.txt & 32768 & 262,144 & 165,508 & 229,376 & 168,711 & 151,224 \\
\hline 8) paper 6.txt & 38105 & 304,840 & 192,182 & 266,735 & 195,599 & 186,408 \\
\hline 9) paper 3.txt & 46526 & 372,208 & 218,195 & 325,682 & 226,239 & 191,328 \\
\hline 10) paper 1.txt & 53161 & 425,288 & 266,692 & 372,127 & 272,009 & 249,400 \\
\hline 11) test 7.txt & 65536 & 524,288 & 304,480 & 458,752 & 314,980 & 262,344 \\
\hline 12) paper 2.txt & 82199 & 657,592 & 380,918 & 575,393 & 397,497 & 333,312 \\
\hline 13) trans.txt & 93695 & 749,560 & 507,249 & 641,438 & 517,973 & 396,528 \\
\hline 14) bib.txt & 111,261 & 890,088 & 582,085 & 778,827 & 591,827 & 430,752 \\
\hline 15) test 8.txt & 131,072 & $1,048,576$ & 621,241 & 917,504 & 650,108 & 561,960 \\
\hline 16) test 9.txt & 262,144 & $2,097,152$ & $1,264,664$ & $1,835,008$ & $1,334,646$ & $1,142,976$ \\
\hline 17) news.txt & 377,109 & $3,016,872$ & $2,312,572$ & $2,639,763$ & $1,998,063$ & $1,862,464$ \\
\hline 18) test 10.txt & 524,288 & $4,194,304$ & $2,533,927$ & $3,670,016$ & $3,140,471$ & $2,385,400$ \\
\hline 19) book 2.txt & 610,856 & $4,886,848$ & $2,946,397$ & $3,817,240$ & $2,980,735$ & $2,772,232$ \\
\hline 20) book 1.txt & 768,771 & $6,150,168$ & $3,564,655$ & $5,381,397$ & $3,605,872$ & $3,126,448$ \\
\hline 21) test 11.txt & $1,048,576$ & $8,388,608$ & $4,748,053$ & $7,340,032$ & $4,887,941$ & $4,233,840$ \\
\hline Average & $204,274.5238$ & $1,634,196.1$ & $991,894.62$ & $1,407,291.8$ & $1,024,210.33$ & $881,510.857$ \\
\hline
\end{tabular}

\section{Appendix B}

Table A2. Compression ratio.

\begin{tabular}{lcccccc}
\hline File name & $\begin{array}{c}\text { Original } \\
\text { size } \\
\text { (bytes) }\end{array}$ & $\begin{array}{c}\text { Original } \\
\text { size } \\
(\text { bits })\end{array}$ & $\begin{array}{c}\text { Huffman } \\
\text { ratio } \\
(\%)\end{array}$ & $\begin{array}{c}\text { FLC } \\
\text { ratio } \\
(\%)\end{array}$ & $\begin{array}{c}\text { HFLC } \\
\text { ratio } \\
(\%)\end{array}$ & $\begin{array}{c}\text { LZW } \\
\text { ratio } \\
(\%)\end{array}$ \\
\hline 1) test 1.txt & 1024 & 8192 & 66.6870 & 87.50 & 68.1762 & 92.4804 \\
2) test 2.txt & 2048 & 16384 & 61.1328 & 75.00 & 62.0239 & 74.8535 \\
3) test 3.txt & 4096 & 32768 & 64.1815 & 87.50 & 65.3289 & 71.6796 \\
4) test 4.txt & 8192 & 65536 & 61.3388 & 87.50 & 62.1032 & 59.2773 \\
5) paper 5.txt & 11,954 & 95632 & 62.1601 & 87.50 & 63.5969 & 58.7 \\
6) test 5.txt & 16,384 & 131072 & 64.7087 & 87.50 & 66.4314 & 66.2231 \\
7) test 6.txt & 32,768 & 262144 & 63.1362 & 87.50 & 64.3581 & 57.6873 \\
\hline
\end{tabular}


Continued

\begin{tabular}{lcccccc}
\hline 8) paper 6.txt & 38,105 & 304840 & 63.0435 & 87.50 & 64.1644 & 61.1494 \\
9) paper 3.txt & 46,526 & 372208 & 58.6217 & 87.50 & 60.7829 & 51.4035 \\
10) paper 1.txt & 53,161 & 425288 & 62.7085 & 87.50 & 63.9587 & 58.6426 \\
11) test 7.txt & 65,536 & 524288 & 58.0749 & 87.50 & 60.0776 & 50.0381 \\
12) paper 2.txt & 82,199 & 657592 & 57.9261 & 87.50 & 60.4473 & 50.6867 \\
13) trans.txt & 93,695 & 749560 & 69.1949 & 87.50 & 70.6578 & 52.9014 \\
14) bib.txt & 111,261 & 890,088 & 65.3963 & 87.50 & 66.4908 & 48.3943 \\
15) test 8.txt & 131,072 & $1,048,576$ & 59.2461 & 87.50 & 61.9991 & 53.5926 \\
16) test 9.txt & 262,144 & $2,097,152$ & 60.3038 & 87.50 & 63.6408 & 54.5013 \\
17) news.txt & 377,109 & $3,016,872$ & 76.6546 & 87.50 & 66.2296 & 61.7349 \\
18) test 10.txt & 524,288 & $4,194,304$ & 60.4135 & 87.50 & 74.8746 & 56.8723 \\
19) book 2.txt & 610,856 & $4,886,848$ & 60.2923 & 87.50 & 68.3253 & 56.7284 \\
20) book 1.txt & 768,771 & $6,150,168$ & 57.9603 & 87.50 & 58.6304 & 50.8351 \\
21) test 11.txt & $1,048,576$ & $8,388,608$ & 56.6012 & 87.50 & 58.2688 & 50.4713 \\
\hline
\end{tabular}

\section{Appendix C}

Table A3. Compression time.

\begin{tabular}{|c|c|c|c|c|c|c|}
\hline File name & $\begin{array}{l}\text { Original } \\
\text { size } \\
\text { (bytes) }\end{array}$ & $\begin{array}{c}\text { Original } \\
\text { size } \\
\text { (bits) }\end{array}$ & $\begin{array}{l}\text { Huffman } \\
\text { time } \\
\text { (ms/char) }\end{array}$ & $\begin{array}{c}\text { FLC } \\
\text { time } \\
\text { (ms/char) }\end{array}$ & $\begin{array}{c}\text { HFLC } \\
\text { time } \\
\text { (ms/char) }\end{array}$ & $\begin{array}{c}\text { LZW } \\
\text { time } \\
\text { (ms/char) }\end{array}$ \\
\hline 1) test 1.txt & 1024 & 8192 & 0.9765 & 0.9765 & 0.9765 & 0.0585 \\
\hline 2) test 2.txt & 2048 & 16,384 & 0.4882 & 0.4882 & 0.4882 & 0.0244 \\
\hline 3) test 3.txt & 4096 & 32,768 & 0.2441 & 0.2441 & 0.2441 & 0.0122 \\
\hline 4) test 4.txt & 8192 & 65,536 & 0.12207 & 0.12207 & 0.2441 & 0.0134 \\
\hline 5) paper 5.txt & 11,954 & 95,632 & 0.0836 & 0.0836 & 0.0836 & 0.0092 \\
\hline 6) test 5.txt & 16,384 & 131,072 & 0.06103 & 0.06103 & 0.12207 & 0.0067 \\
\hline 7) test 6.txt & 32,768 & 262,144 & 0.0305 & 0.0305 & 0.0610 & 0.0067 \\
\hline 8) paper 6.txt & 38,105 & 304,840 & 0.0262 & 0.0262 & 0.0524 & 0.0057 \\
\hline 9) paper 3.txt & 46,526 & 372,208 & 0.0214 & 0.0214 & 0.0429 & 0.0058 \\
\hline 10) paper 1.txt & 53,161 & 425,288 & 0.0188 & 0.0188 & 0.0376 & 0.0052 \\
\hline 11) test 7.txt & 65,536 & 524,288 & 0.01525 & 0.01525 & 0.0305 & 0.005 \\
\hline 12) paper 2.txt & 82,199 & 657,592 & 0.0121 & 0.0243 & 0.0364 & 0.0046 \\
\hline 13) trans.txt & 93,695 & 749,560 & 0.0218 & 0.0218 & 0.0327 & 0.00469 \\
\hline 14) bib.txt & 111,261 & 890,088 & 0.0179 & 0.0179 & 0.0269 & 0.00494 \\
\hline 15) test 8.txt & 131,072 & $1,048,576$ & 0.0076 & 0.0076 & 0.0152 & 0.0045 \\
\hline 16) test 9.txt & 262,144 & $2,097,152$ & 0.0038 & 0.0076 & 0.0114 & 0.0041 \\
\hline 17) news.txt & 377,109 & $3,016,872$ & 0.0079 & 0.0079 & 0.0132 & 0.00392 \\
\hline 18) test 10.txt & 524,288 & $4,194,304$ & 0.0019 & 0.00653 & 0.0076 & 0.00398 \\
\hline 19) book 2.txt & 610,856 & $4,886,848$ & 0.0065 & 0.0065 & 0.0110 & 0.00386 \\
\hline 20) book 1.txt & 768,771 & $6,150,168$ & 0.0230 & 0.0230 & 0.0345 & 0.00386 \\
\hline 21) test 11.txt & $1,048,576$ & $8,388,608$ & 0.0019 & 0.0028 & 0.0038 & 0.00387 \\
\hline
\end{tabular}




\section{Appendix D}

Table A4. Entropy.

\begin{tabular}{|c|c|c|c|c|c|c|}
\hline File name & $\begin{array}{l}\text { Original } \\
\text { size } \\
\text { (bytes) }\end{array}$ & $\begin{array}{l}\text { Original } \\
\text { size } \\
\text { (bits) }\end{array}$ & $\begin{array}{c}\text { Huffman } \\
\text { entropy } \\
\text { (bits/char) }\end{array}$ & $\begin{array}{c}\text { FLC } \\
\text { entropy } \\
\text { (bits/char) }\end{array}$ & $\begin{array}{c}\text { HFLC } \\
\text { entropy } \\
\text { (bits/char) }\end{array}$ & $\begin{array}{c}\text { LZW } \\
\text { entropy } \\
\text { (bits/char) }\end{array}$ \\
\hline 1) test 1.txt & 1024 & 8192 & 5.3349 & 7.00 & 5.4541 & 7.3984 \\
\hline 2) test 2.txt & 2048 & 16,384 & 4.8906 & 6.00 & 4.9619 & 5.9882 \\
\hline 3) test 3.txt & 4096 & 32,768 & 5.1345 & 7.00 & 5.2263 & 5.7343 \\
\hline 4) test 4.txt & 8192 & 65,536 & 4.9071 & 7.00 & 4.9682 & 4.7421 \\
\hline 5) paper 5.txt & 11,954 & 95,632 & 4.9728 & 7.00 & 5.0877 & 4.696 \\
\hline 6) test 5.txt & 16,384 & 131,072 & 5.1766 & 7.00 & 5.3145 & 5.2978 \\
\hline 7) test 6.txt & 32,768 & 262,144 & 5.0509 & 7.00 & 5.1486 & 4.6149 \\
\hline 8) paper 6.txt & 38,105 & 304,840 & 5.0434 & 7.00 & 5.1331 & 4.8919 \\
\hline 9) paper 3.txt & 46,526 & 372,208 & 4.6897 & 7.00 & 4.8626 & 4.1122 \\
\hline 10) paper 1.txt & 53,161 & 425,288 & 5.0166 & 7.00 & 5.1167 & 4.6914 \\
\hline 11) test 7.txt & 65,536 & 524,288 & 4.6459 & 7.00 & 4.8062 & 4.003 \\
\hline 12) paper 2.txt & 82,199 & 657,592 & 4.6341 & 7.00 & 4.8357 & 4.0549 \\
\hline 13) trans.txt & 93,695 & 749,560 & 5.5355 & 7.00 & 5.6526 & 4.2321 \\
\hline 14) bib.txt & 111,261 & 890,088 & 5.2317 & 7.00 & 5.3192 & 3.8715 \\
\hline 15) test 8.txt & 131,072 & $1,048,576$ & 4.7396 & 7.00 & 4.9599 & 4.2874 \\
\hline 16) test 9.txt & 262,144 & $2,097,152$ & 4.8243 & 7.00 & 5.0912 & 4.3601 \\
\hline 17) news.txt & 377,109 & $3,016,872$ & 6.1323 & 7.00 & 5.2983 & 4.9387 \\
\hline 18) test 10.txt & 524,288 & $4,194,304$ & 4.8330 & 7.00 & 5.9899 & 4.5497 \\
\hline 19) book 2.txt & 610,856 & $4,886,848$ & 4.8233 & 7.00 & 5.4660 & 4.5382 \\
\hline 20) book 1.txt & 768,771 & $6,150,168$ & 4.6368 & 7.00 & 4.6904 & 4.0668 \\
\hline 21) test 11.txt & $1,048,576$ & $8,388,608$ & 4.5281 & 7.00 & 4.6615 & 4.0377 \\
\hline Sum & $4,289,765$ & $34,318,120$ & 104.7817 & 146 & 108.0446 & 99.1073 \\
\hline avg. & $204,274.52$ & $1,634,196$ & 4.9896048 & 6.952380952 & 5.144980952 & 4.719395238 \\
\hline
\end{tabular}

\section{Appendix E}

Table A5. Average ratio.

\begin{tabular}{lcccccc}
\hline $\begin{array}{l}\text { Algorithm } \\
\text { name }\end{array}$ & $\begin{array}{c}\text { Average } \\
\text { original } \\
\text { size (bytes) }\end{array}$ & $\begin{array}{c}\text { Average } \\
\text { original } \\
\text { size (bits) }\end{array}$ & $\begin{array}{c}\text { Average } \\
\text { compress } \\
\text { size (bits) }\end{array}$ & $\begin{array}{c}\text { Average } \\
\text { compress } \\
\text { ratio (\%) }\end{array}$ & $\begin{array}{c}\text { Average } \\
\text { time } \\
\text { (ms/char) }\end{array}$ & $\begin{array}{c}\text { Average } \\
\text { entropy } \\
\text { (bits/char) }\end{array}$ \\
\hline 1) Huffman & $204,274.5238$ & $1,634,196.19$ & $991,894.62$ & 60.69618 & 0.10438 & 4.85569 \\
2) FLC & & $1,407,291.81$ & 86.11523 & 0.105408 & 6.8892 \\
3) HFLC & & $1,024,210.33$ & 62.67364 & 0.12265 & 5.01389 \\
4) LZW & & $881,510.857$ & 58.9930047 & 0.00929429 & 4.719395238 \\
\hline
\end{tabular}

